\title{
PAMELA - A MODEL FOR AN FFAG BASED HADRON THERAPY MACHINE
}

\author{
K. Peach, J. Cobb, T. Yokoi, JAI, Oxford, UK, Ian Gardner, STFC/RAL/ISIS, Chilton, UK, Rob \\ Edgecock STFC/RAL/PPD, Chilton, UK, Mike Poole STFC/ASTeC, Daresbury UK, J. Pozimski, \\ Imperial College, London, UK, Bob Cywinski, Leeds University, UK, Bleddyn Jones, Birmingham \\ University, UK, Gilles McKenna, Oxford University, UK, Boris Vojnovic, Melvyn Folkard, Grey \\ Cancer Institute, London, UK, Karen Kirkby, Roger Webb, Surrey University, UK, Roger Barlow, \\ Manchester University, UK, Alex Elliott, Beatson Oncology Centre, Glasgow, UK.
}

\begin{abstract}
Approximately one third of the world's 15000 accelerators are used for tumour therapy and other medical applications [1]. The characteristics of FFAGs make them ideally suited to such applications, as the much smaller magnet size and greater compactness offers considerable cost and operational benefits. In the first stage the work on PAMELA will focus on the optimization of the FFAG design to deliver the specific machine parameters demanded by therapy applications. In this phase of the PAMELA project the effort will concentrate on the design of a semi-scaling type FFAGs to deliver a $450 \mathrm{MeV} / \mathrm{u}$ carbon ion beam, including detailed lattice and tracking studies. The second stage will use the existing expertise in the BASROC consortium [2] to undertake a design of the magnets and RF system for PAMELA. An outline of the overall concept of PAMELA will be discussed and the actual status of the work will be presented.
\end{abstract}

\section{INTRODUCTION}

There has also been a recent, very strong interest in using NS-FFAGs for cancer therapy, as the much smaller magnet size and greater compactness would add both cost and operational benefits, in particular the ease of delivering a beam at any energy. Some initial simulation work has been done, the most advanced by a small collaboration led by the Brookhaven National Laboratory [3]. This envisages a proton and carbon ion complex consisting of an RFQ and short linac, and then three rings. Both proton and carbon ions are accelerated in the linac. The protons are then accelerated to 31 and $250 \mathrm{MeV}$ in the first two rings and carbon to 69 and $400 \mathrm{MeV} / \mathrm{u}$ in the second and third. The rings are 35,43 and $52 \mathrm{~m}$ in circumference, respectively, and the largest horizontal magnet aperture, in the third ring, is less than $40 \mathrm{~mm}$.

While the main properties of FFAG's are ideal suited for medical applications there are still some serious issues to be solved before building such a machine. The main challenge for the use of NS-FFAG is the resonance crossing acceleration. The lattice of NS-FFAG usually consists of linear magnet. The simplicity and flexibility coming from this are the advantages of NS-FFAG. However, due to the nature of a linear lattice, it inevitably encounters many resonances during acceleration. Nevertheless, there is considerable concern that the resonances will have a much too severe effect on the beam to make this a viable option. This will be investigated by EMMA, which is to build a $20 \mathrm{MeV}$ electron NS-FFAG as a proof-of-principle machine [5].

In PAMELA, the accelerated beams are non-relativistic proton or heavy ions. Therefore, though the feasibility of NS-FFAG with resonance crossing acceleration is established in EMMA, considerable additional design work is required especially for lattice design and acceleration scheme in PAMERA, where the acceleration process is much slower compared with EMMA. Additionally the problem of beam injection and extraction into and from the different rings is another challenge in PAMELA.

The first stage of this work will be the design of machines to deliver a $450 \mathrm{MeV} / \mathrm{u}$ carbon ion beam with small or zero tune variations. This will include detailed lattice and tracking studies. The second phase will be to use the existing expertise in the consortium to undertake a design of the magnets and RF system for this machine. The output from these stages will be a demonstration of feasibility. The third phase will be a preliminary cost estimate for the complex, allowing comparison with existing technologies on performance and cost.

The next stage will be to take the work done and to scale the design down to both $70 \mathrm{MeV}$ and $230 \mathrm{MeV}$ proton machines as possible prototypes, including cost estimates. The second of these machines would be preferable as not only could it be developed into a therapy machine, it could also be used to produce $68 \mathrm{MeV} / \mathrm{u}$ carbon ions.

As there is a lack of evidence demonstrating the benefits of carbon over protons, this machine would be used to "treat" mice to obtain this. In addition, it would provide scientifically obtained evidence demonstrating the benefits of hadron therapy over standard radiotherapy. If the higher energy machine proves to be too expensive, however, the lower energy machine could be used both as a NS-FFAG prototype and also to do the comparison between hadron and radiotherapy.

\section{BEAM PARAMETERS REQUIRED FOR A CANCER THERAPY MACHINE}

In a first step, a list of beam parameters as required for a hadron therapy machine has been discussed and agreed on. The beam energy of the protons should be tuneable in 
the range between 50 and $270 \mathrm{MeV}$ (up to $325 \mathrm{MeV}$ for proton radiography) and for carbon a beam energy up to $450 \mathrm{MeV} /$ nucleon is required.

For the treatment with protons a dose of 2 Gray/minute in a volume of 2 litres (protons) will be the goal. One should note that the dose given refers to the radiation deposited in the tumour. The dose is (almost) independent of the incident energy of the proton. The residual range when the Bragg peak starts is about $5 \mathrm{~cm}$. Taking the irradiated tissue as water, this corresponds in the standard "Range/Mass versus Momentum/Mass" tables to a $\beta \gamma$ of about 4 , or a proton momentum of about $0.3 \mathrm{GeV} / \mathrm{c}$, or a proton energy of around $45 \mathrm{MeV}$. Taking this into account a dose of 2 Gray/minute would require $\sim 1.35 \times$ $10^{13} \mathrm{MeV}$ of proton energy deposited per minute at 45 $\mathrm{MeV} /$ proton. This gives a proton flux of $3 \times 10^{11}$ protons per second, or a average current of $50 \mathrm{nA}$.

The requirement for the dose calibration is that the dose deposition in a $5 \mathrm{~mm} \times 5 \mathrm{~mm} \times 5 \mathrm{~mm}$ cube $\left(125 \mathrm{~mm}^{3}\right)$ is controlled to within 5\%. This implies (assuming 100 pulses) that the individual pulses are controlled and known at the level of $50 \%$, which is a major advantage of an rapid cycling machine for such purposes. The maximum field to be irradiated should be of $40 \mathrm{~cm} \times 40$ $\mathrm{cm}$ in size and the penetration depth will be (depending on the beam energy) up to $25 \mathrm{~cm}$. The spot size of the beam in the tumour should be below $1 \mathrm{~cm}$. For raster scanning, the requirement is to perform 60 "spills" in about 2-3 minutes, which is defined by the maximum time the patient can be expected to be "still". This requires changing the penetration depth and therefore the speed of the energy scanning to be at the $\mathrm{Hz}$ level ( $\sim 0.1-1$ $\mathrm{Hz}$ ). The number of energy steps will probably be limited by the machine. From a clinical point of view, the energy steps should be small compared with the size of the Bragg peak (45 MeV see above, so 5-10 MeV). However, the machine is likely to deliver an energy step of a few hundred $\mathrm{keV} / \mathrm{turn}$, so that the granularity of the energy steps should not be of concern.

The energy spread allowed for the machine is related to the straggling of the ions in the tissue. A proton with energy $250 \mathrm{MeV}$ with straggling $\sim 2.5 \%$ has a kinetic energy spread (full width) of about $10-15 \mathrm{MeV}$. If we do not want the energy spread of the machine to dominate over the straggling, we need to keep the energy spread on the beam at $250 \mathrm{MeV}$ to be less than $5 \mathrm{MeV}$, or $2 \%(\sim 4 \%$ on momentum). The main requirements of a hadron therapy machine are summarized in table 1 .

Table 1: Summary of Beam Parameters for an FFAG Based Hadron Cancer Therapy Facility PAMELA

\begin{tabular}{|c|c|c|}
\hline & protons & carbon \\
\hline Beam energy $(\mathrm{MeV} / \mathrm{u})$ & $<325$ & $<450$ \\
\hline Energy step size $(\mathrm{MeV} / \mathrm{u})$ & $\sim 10$ & $\sim 10$ \\
\hline Beam current $(\mathrm{enA})$ & 50 & 3 \\
\hline Particle flux $(1 / \mathrm{sec})$ & $3 \times 10^{11}$ & $3 \times 10^{09}$ \\
\hline
\end{tabular}

\section{THE FRONT END}

Two different scenarios for the Front End are under discussion both with different advantages and disadvantages. While the first one requires no change in the field power of the dipole for operation with both species at the price of a lower proton current, the other needs adjustment of the FFAG dipoles when changing the species making a fast switching difficult.

In the first scenario a commercially available small cyclotron with an output energy of $4 \mathrm{MeV}$ will be used to inject a proton beam into the FFAG (see figure 1). In this first phase this beam could be accelerated to the final energy of $350 \mathrm{MeV}$ using two FFAGs, allowing various oncology studies and proton therapy. In the second phase of the project a carbon injector consisting of an ECR ion source, an LEBT utilizing a spectrometer for mass/charge separation and an RFQ (beam energy at the output of the RFQ will be $1 \mathrm{MeV} / \mathrm{u}$, see second scenario) will be added together with a third FFAG to allow treatment with carbon ions up to an energy of $450 \mathrm{MeV}$.

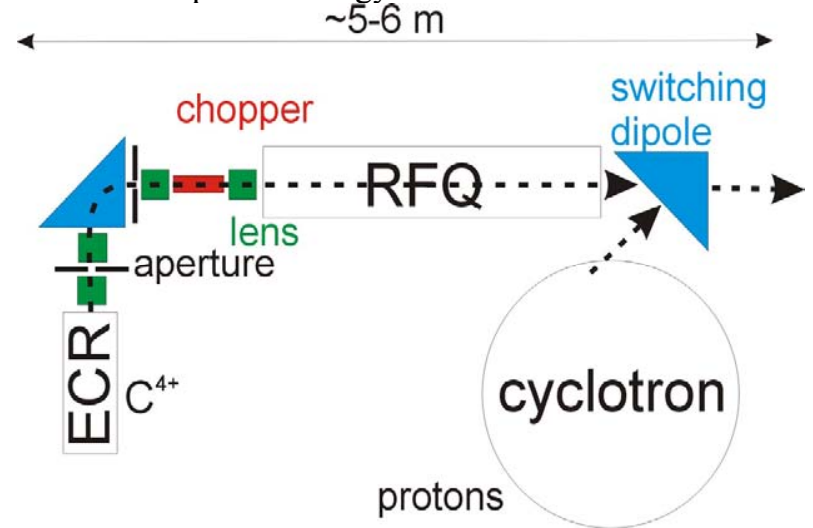

Figure 1: Schematic drawing of the beam injector scenario 1.

The second scenario is shown in figure 2. It will consist in the first phase of an ECR ion source delivering a beam current of $2 \mathrm{~mA}$ of protons with $10 \mathrm{keV}$ beam energy, in the second phase an additional ECR source will be added to deliver $0.2 \mathrm{~mA}$ of $\mathrm{C}^{4+}$ at $120 \mathrm{keV}$ beam energy. The beam is injected into LEBT section consisting of solenoids for beam focussing and set up as a spectrometer line utilizing a magnetic dipole for charge/mass separation followed by an RFQ running at about 200 $\mathrm{MHz}$.

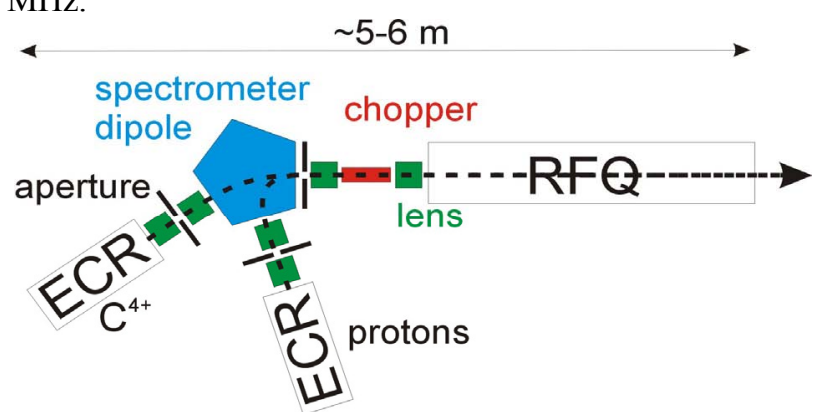

Figure 2: Schematic drawing of the beam injector scenario 2 . 
The RFQ itself will be approximately $2.5 \mathrm{~m}$ long and requires $300 \mathrm{~kW}$ of RF power. It will accelerate the beam to an energy of $1 \mathrm{MeV} / \mathrm{u}$. The beam parameters at RFQ output are summarized in Table 2.

Table 2: Output Parameters for Beam Injector Scenario 1 and 2

\begin{tabular}{|c|c|c|}
\hline & Protons scenario 1 (2) & $\mathrm{C}^{4+}$ \\
\hline peak Current (mA) & $<0.1(>2)$ & $\sim 0.2$ \\
\hline$\varepsilon_{100 \%, \mathrm{n}}\left(\pi^{*} \mathrm{~mm}^{*} \mathrm{mrad}\right)$ & $0.4-0.6$ & \\
\hline$\varepsilon_{\text {RMS }, \mathrm{n}}\left(\pi^{*} \mathrm{~mm} * \mathrm{mrad}\right)$ & $0.1-0.15$ & \\
\hline RF Frequency $(\mathrm{MHz})$ & $\sim 200$ & \\
\hline Bunch length $(\mathrm{ps})$ & $\sim 500$ & \\
\hline Pulse length (ms) & $\sim 100$ & \\
\hline Repetition rate $(\mathrm{Hz})$ & $\sim 1000$ & \\
\hline Particles per bunch \# & $10^{6}-10^{7}\left(10^{7}-10^{8}\right)$ & $10^{5}-10^{6}$ \\
\hline Beam energy (MeV/u) & $4(1)$ & 1 \\
\hline
\end{tabular}

Behind the RFQ a MEBT beam line consisting of RF bunching cavities and magnetic quadrupoles for transversal and longitudinal beam focussing to adopt the RFQ output parameters to the required FFAG injection parameters. Additionally a stripping foil will be used to increase the charge state of the carbon ions from $4^{+}$to $6^{+}$ to increase the acceleration efficiency in the FFAG rings. The position of the stripping foil is still under discussion but between the first and second FFAG is most likely, as the stripping efficiency will be much higher compared with stripping behind the RFQ.

\section{FFAG LAYOUT}

One of the advantages of NS-FFAG for a particle therapy facility is flexibility in facility operation. Due to the fixed field, just by changing the injection particle, it can immediately change the delivering particle.

The planned facility aims to deliver proton and heavy ion beam for treatment. Thus, considering the injector energy and reasonable momentum range of one NSFFAG ring, which is typically about factor of 3 , the facility is to consist of three cascaded rings. The beam parameters are summarized in Table 3 . The second ring works as the final ring for proton therapy and the booster for the final carbon ring as well.

\section{BEAM DELIVERY AND GANTRY}

The fast beam extraction of a FFAG accelerator allows it to deliver beams to multiple ports simultaneously. It is a unique feature of FFAG as an accelerator for particle therapy and it drastically improves efficiency of a treatment facility. The beam structure is pulsed one, which is well fitted to the spot scanning irradiation, but it needs consideration in employing the board beam with a wobbler.

Table 3: Parameters of Accelerator Complex

\begin{tabular}{|l|l|l|l|}
\hline $\begin{array}{l}\text { Proton } \\
\text { Injector 1 }\end{array}$ & $\begin{array}{l}\text { Injection } \\
(\mathrm{MeV})\end{array}$ & $\begin{array}{l}\text { Extraction } \\
(\mathrm{MeV}, \mathrm{max})\end{array}$ & $\begin{array}{l}\text { Momentum } \\
\text { ratio }\end{array}$ \\
\hline 1st ring & 4 & 40 & 3.2 \\
\hline 2nd ring & 40 & 350 & 3.2 \\
\hline $\begin{array}{l}\text { Proton } \\
\text { Injector 2 }\end{array}$ & & & \\
\hline 1st ring & 1 & 10 & 3.2 \\
\hline 2nd ring & 10 & 100 & 3.2 \\
\hline 3rd ring & 100 & 350 & 1.9 \\
\hline Carbon & & & \\
\hline 1st ring & 1 & 10 & 3.2 \\
\hline 2nd ring & 10 & 100 & 3.2 \\
\hline 3rd ring & 100 & 450 & 3.1 \\
\hline
\end{tabular}

For the rotating gantry, an ordinary gantry as developed for existing facilities can be used as long as the energy changing rate is slow (typically several times/sec) However, if a much faster change in energy, for instance as fast as the repetition rate of FFAG, is required, another approach such as FFAG gantry proposed by [6] must be taken.

\section{SUMMARY}

While the PAMELA project has just started and the team is still under formation, the main parameters required to built a machine for oncology studies as a first step, and to extend this machine finally to a full hadron cancer therapy machine based on 3 FFAGs, have been defined. Within the 3 years of the study a robust design based also on the experiences gained from the commissioning of EMMA is expected.

\section{REFERENCES}

[1] "How Particles can be therapeutic", Physics World, August 2003. http://physicsweb.org/articles/world/ $16 / 8 / 9$

[2] BASROC website http://www.basroc.org.uk

[3] C. Johnstone, W. Wan and A. Garren "Fixed Field Circular Accelerator Designs", Proc. PAC'99, New York, 1999, http://www.jacow.org

[4] CONFORM website http://www.conform.ac.uk

[5] R. Edgecock, "EMMA - the World's First Nonscaling FFAG", these proceedings

[6] E.Keil, A.M..Sessler, D.Trbojevic, Proceedings of EPAC2006, p. 1681, http://www.jacow.org 